\title{
Associação entre História de Aleitamento e Relatos de Hábitos Orais e Alergia em Crianças
}

Association between History of Breastfeeding and Reports of Oral Habits and Allergy in Children

Juliane Priscila Brustolin ${ }^{1}$, Débora Martini Dalpian², Fabricio Batistin Zanatta ${ }^{3}$, Luciano Casagrande ${ }^{4}$

\section{Abstract}

Aim: The purpose of this study was to assess the association between type and duration of breastfeeding with the presence of mouth breathing habits, bruxism, anterior open bite and presence of respiratory allergic conditions. Materials and Methods: It was analyzed all dental records of children aging between 0 and 12 years old who were attended by dental students Franciscan University Center (UNIFRA) since 2005 to 2010. Of a total of 507 dental records, 395 were included in the study. Results: The results showed an association between breastfeeding the presence of mouth breathing, bruxism and anterior open bite $(p<0.05)$. However, there was no association between breast feeding and the presence of respiratory allergy as well as time of breastfeeding and the presence of mouth breathing, bruxism, and anterior open bite allergy. The majority of children UNIFRA received more natural breastfeeding (85\%) than artificial (14\%). Conclusion: The breast feeding provided the baby can contribute to the proper development of the face and thus prevent the emergence of changes in the stomatognathic system.

Keywords: Breastfeeding. Bruxism. Hypersensitivity. Mouth breathing. Openbite.

\section{Resumo}

Objetivo: Este trabalho teve como propósito verificar a associação entre o tipo e o tempo de amamentação fornecida ao bebê com a presença de hábitos de respiração bucal, bruxismo, mordida aberta anterior e presença de processos respiratórios alérgicos. Materiais e métodos: Foram analisados todos os prontuários clínicos de crianças entre 0 a 12 anos de idade atendidos nos ambulatórios odontológicos do Centro Universitário Franciscano (UNIFRA), no período de 2005 a 2010. De um total de 507 prontuários, foram incluídos no estudo prontuários de 395 crianças. Resultados: Os resultados demonstraram haver associação entre o tipo de amamentação com a presença de respiração bucal, bruxismo e mordida aberta anterior $(p<0,05)$. No entanto, não foram encontradas associações entre o tipo de amamentação fornecida ao bebê e a presença de processos respiratórios alérgicos, bem como o período de amamentação e a presença de hábitos de respiração bucal, bruxismo, mordida aberta anterior e alergia. Alem disso, constatou-se que a maioria das crianças atendidas nos ambulatórios odontológicos da UNIFRA recebeu mais amamentação natural (85\%) que artificial (14\%). Conclusão: Concluiu-se, portanto, que o tipo de amamentação fornecida ao bebê pode colaborar para o correto desenvolvimento da face e assim evitar o surgimento de alterações no sistema estomatognático.

Descritores: Amamentação. Bruxismo. Alergia. Respiração bucal. Mordida aberta.
${ }^{1}$ Cirurgiã Dentista. Mestre em Odontopediatria pela UFRGS.

${ }^{2}$ Mestre em Odontopediatria. Professora do Centro Universitário Franciscano. Santa Maria, Brasil

${ }^{3}$ Doutor em Periodontia. Professor da Universidade Federal de Santa Maria. Santa Maria, Brasil.

${ }^{4}$ Doutor em Odontopediatria. Professor da Universidade Federal do Rio Grande do Sul. Porto Alegre, Brasil.

Correspondência: Juliane Priscila Brustolin

Endereço: Avenida Coronel Lucas de Oliveira. № 1853, Apt 404 - Bairro Petrópolis Porto Alegre, RS - Brasil

E-mail: julianebrustolin@yahoo.com.br

Data de Submissão: $26 / 06 / 2012$

Data de Aceite: 04/03/2013

\section{Introdução}

Apesar das inúmeras vantagens que o aleitamento natural proporciona ao bebê a amamentação deixou de ser vital ao ser humano a partir dos últimos 150-300 anos, com o avanço da industrialização. Esta trouxe a possibilidade de sobrevivência das crianças sem mamar no peito, na medida em que este foi substituído pelo uso de alimentos processados para compensar deficiências fisiológicas maternas ou da própria criança (PRAETZEL, 1997). Esse processo foi influenciado por fatores como o estilo de vida moderna, a personalidade da mãe, a história pessoal e a influência da sociedade que direcionam a decisão da mãe para 0 aleitamento artificial(PRAETZEL et al., 1997; CZERNAY; BOSCO, 2003). De acordo com relatos maternos, dentre as razões da interrupção do aleitamento natural estão a hipogalactia da puérpera, a utilização de drogas para o controle de hipertensão e distúrbios psicológicos, a mastite, os bebês com dificuldades de sucção, problemas relacionados ao ciúme do filho mais velho (MIZUNO et al., 2004), o ingurgitamento mamário, a produção de leite interrompida por razão emocional, o comprometimento da saúde da mãe e da própria criança, como por exemplo, a contaminação do lactente pelo vírus HIV (VINHA et al.,2002).

Contudo, segundo orientações da Organização Mundial de Saúde, as mães devem fornecer o leite materno de forma exclusiva durante os primeiros seis meses de vida de uma criança, já que na sua composição estão presentes proteínas, gorduras, carboidratos, vitaminas e anticorpos essenciais para uma boa formação da imunidade. Além disso, o ato fisiológico da sucção da mama estimula a função gástrica normal do bebê, possui ação psicológica calmante pelo contato materno e calor do corpo da mãe, evita a superalimentação e diminui a deglutição de ar(MOREIRA; CORREIA, 1998).Pode também exercer importante papel preventivo nas alterações mio funcionais e ortodônticas, promovendo um correto crescimento e desenvolvimento do Sistema Estomatognático (BALDRIGHI et al., 2001; MOHEBBI et al., 2008; PERES et al., 2007; PRAETZEL et al, 2002; SCAVONE JUNIOR et al., 2008; VÁSQUEZNAVA et al., 2008). 
Ferreira e Toledo(1997) avaliaram a associação entre o tempo de aleitamento materno e a etiologia de alguns hábitos bucais perniciosos em pré-escolares, tendo observado que crianças cuja utilização de mamadeira foi anterior às demais adquiriram mais hábitos bucais nocivos quando comparadas as que prolongaram a amamentação natural além do primeiro trimestre de vida. Ademais modificações iniciais na fonação, deglutição, respiração bucal, sucção de dedo e de chupeta parecem ser sinais clínicos iniciais de futuras más-oclusões, sendo o padrão de disfunção Miofuncional relacionado à freqüência, intensidade e duração do hábito que a criança pratica(QUELUZ; GIMENEZ, 2000).

Sendo assim, dada a importância do aleitamento materno no desenvolvimento da criança o presente estudo verificou as associações de maloclusões, bruxismo, respiração bucal e alergia com o tempo e tipo de amamentação fornecida ao bebê.

\section{Materiais e Métodos}

A partir de uma coleta transversal foram analisados todos os prontuários clínico-odontológicos de pacientes com idades entre 0-12 anos, de ambos os sexos, que estiveram sob atendimento odontológico nos Ambulatórios do Centro Universitário Franciscano (UNIFRA) no período de 2005 a 2010. A UNIFRA é uma escola privada de Odontologia situada na cidade de Santa Maria - RS, que realiza atendimento à população local, estando entre os locais de maior atendimento populacional da Região Central do Estado. Todos os exames de diagnóstico clínico, assim como o preenchimento dos prontuários, foram realizados por alunos da graduação e revisados pelos professores da instituição. Ainda, os dados anamnéticos coletados dos pacientes desta faixa etária foram obtidos por entrevista às mães ou responsáveis.

Informações pertinentes à anamnese como o período gestacional, história médica passada e atual, assim como informações coletadas durante o exame clínico odontológico foram coletadas por dois avaliadores previamente treinados. No entanto, 112 prontuários incompletos ou mal preenchidos de um total de 507 foram considerados não elegíveis e, portanto, excluídos da análise não sendo contabilizados na amostra final.

\section{Análise dos Dados}

Associações entre o tipo de amamentação fornecido ao bebê (natural ou artificial) e o período de duração desta com a presença de alergia, hábitos de respiração bucal, bruxismo e mordida aberta anterior foram testadas de uma forma dicotômica.

A identificação dos pacientes com processos alérgicos respiratórios foi realizada através de informações obtidas pelos pais ou responsáveis. Já os pacientes portadores de respiração predominantemente bucal foram avaliados não somente pelo relato dos responsáveis como também, pelo perfil facial alterado (alongado), musculatura facial hipotônica, lábios entreabertos, palato ogival, protrusão da arcada superior, olhos inclinados para baixo e presença de olheira e fluxo de ar nasal. O mesmo procedeu com as crianças portadoras de bruxismo onde o relato dos responsáveis foi comprovado com a presença de hipertonicidade muscular e também desgastes dentários acentuados predominantemente nas faces incisal e oclusal dos dentes, não compatíveis com o desgaste fisiológico.

Foi definida amamentação predominantemente natural aquelas crianças que receberam somente leite materno durante 0 período de 6 meses já que este é também preconizado como período ideal de amamentação pela Organização Mundial de Saúde. No entanto, para amamentação artificial foram classificados os pacientes que receberam nutrição por meios adicionais como mamadeira, desde os dois primeiros meses de vida.
Utilizou-se o teste estatístico Qui - Quadrado com o auxílio do programa estatístico Portable PASW Statistics 18- SPSS.

Este projeto teve autorização do Comitê de Ética e pesquisa da Instituição. Registro na CONEP $n^{\circ} \cong 1246$. Registro CEP/UNIFRA: 177.2011.2.

\section{Resultados}

Após a análise de 507 prontuários clínicos, 395 foram considerados elegíveis. De uma forma geral, foi observado que aproximadamente $85 \%$ das crianças avaliadas receberam amamentação predominantemente natural e $15 \%$ amamentação artificial.

A análise dos dados demonstrou haver associação entre o tipo de amamentação fornecida ao bebê com a presença de hábito de respiração bucal $(p=0.004)$, bruxismo $(p=0.003)$ e mordida aberta anterior $(p=0.001)$. Contudo, não foi encontrada associação entre 0 tipo de amamentação com presença de alergia $(p=0.07)$.

Não foi observada associação entre o tempo de amamentação com a presença de hábitos como respiração bucal $(p=0.157)$, bruxismo $(p=0.393)$, mordida aberta anterior $(p=0.042)$ e alergia $(p=0.961)$.

Estes dados podem ser confirmados na Tabela 1.

Tabela 1. Correlação entre tempo e tipo de amamentação com presença de respiração bucal, bruxismo e mordida aberta anterior.

\begin{tabular}{lccccccc} 
& \multicolumn{2}{c}{$\begin{array}{c}\text { Respirador } \\
\text { bucal }\end{array}$} & \multicolumn{2}{c}{ Bruxismo } & \multicolumn{2}{c}{$\begin{array}{c}\text { Mordida aberta } \\
\text { anterior }\end{array}$} \\
\cline { 2 - 8 } & Sim & Não & Sim & Não & Sim & Não \\
\hline $\begin{array}{l}\text { Amamentação } \\
\text { natural }\end{array}$ & 155 & 181 & 109 & 227 & 34 & 247 \\
\hline $\begin{array}{l}\text { Amamentação } \\
\text { artificial }\end{array}$ & 34 & 20 & 28 & 26 & 18 & 20 \\
\hline $\mathrm{p}^{*}$ & 0.004 & & 0.003 & & $<0.001$ \\
\hline
\end{tabular}

\section{Discussão}

O presente estudo avaliou a partir de dados coletados em prontuários de crianças atendidas em uma instituição de ensino superior, as associações entre o tipo e o tempo de aleitamento materno com algumas características do sistema estomatognático. Foi observada associação entre o aleitamento artificial com os desfechos para respiração bucal, bruxismo, e mordida aberta anterior.

Ao nascer, a criança apresenta a mandíbula localizada distalmente em relação à maxila. Com os exercícios de rebaixamento, anteroposteriorização e elevação concomitante da mandíbula durante a ordenha o crescimento ósseo é corretamente estimulado(CARVALHO, 1997; TOLLARA et al., 2001). Quando a criança abocanha a mama, naturalmente fecha a boca, veda os lábios corretamente à língua, estimulando toda a musculatura que trabalha nessas funções, desenvolvendo-as corretamente. Desta forma, apresenta menor chance de desenvolver maus hábitos orais e de várias alterações fonoaudiológicas e odontológicas (PRAETZEL et al., 2002).

Existe a possibilidade de alterações nos padrões normais de respiração ocorrer e a respiração bucal se estabelecer, mesmo em crianças com menos de seis meses de vida. A respiração bucal consiste na adaptação de estruturas faciais envolvidas direta ou 
indiretamente na respiração e que podem levar o indivíduo a manter a boca aberta, determinando uma via mais curta para a entrada do ar (cavidade bucal), que não será filtrado, aquecido, umidificado e pressurizado (funções primariamente nasais) (PRAETZEL et. al., 2002).Ainda, esta condição pode ocorrer devido a processos obstrutivos na região das vias aéreas superiores a qual pode instalarse por vários motivos como, edema da mucosa nasal, pólipos nasais, hipertrofia de cornetos, rinite alérgica, aumento das amígdalas palatinas, desvio de septo e hipertrofia de adenóide(FREITAS et al., 2001; MARCHESAN, 1998; PRATES et al.,1997).

Muitas são as repercussões associadas com respiração bucal, em função adaptação das estruturas faciais, tornando músculos, lábios e língua hipotônicos, ossos com alteração de desenvolvimento, dando à criança uma característica bem peculiar como postura incorreta, ombros caídos e face alongada e estreita. Também pode apresentar deglutição atípica, má oclusão, palato ogival, ronco, olheiras além de apnéia noturna durante o sono (ARAÚJO et al., 2004).

Contudo, autores como Lusvarghi(1999) afirmam que apesar de a respiração bucal ser considerada um desvio, pode funcionar, às vezes, como um padrão de respiração complementar e muitas vezes normal, e que uma pessoa nunca é exclusivamente respiradora bucal: o que existe é a respiração predominantemente bucal ou mista.

Ferreira e Toledo(1997) em um estudo realizado com 427 crianças entre 3 e 6 anos de idade, mostraram que quanto mais prolongado o aleitamento materno, menor a ocorrência de hábitos orais nocivos como a respiração oral. Considerando os nossos dados podemos observar que a amamentação exclusivamente materna provavelmente influenciou na prevenção da respiração predominantemente bucal. No entanto em razão de ser uma alteração causada por diversos fatores como a acima já citado, há uma imensa limitação na construção de um estudo de associação direta entre o efeito da ausência de amamentação natural com síndrome do respirador bucal.

Guerrae Mujica(1999)avaliaram 122 crianças com idade entre 5 e 8 anos e observaram que $77,8 \%$ delas foram amamentadas exclusivamente no peito, porém por um período inadequado, favorecendo o surgimento de hábitos bucais patológicos como o de hiperfunção oral ou bruxismo. Contudo, esta relação não foi observada no presente estudo, já que das 395 crianças avaliadas, 227 não foram amamentadas no seio materno e apresentavam bruxismo. Em contrapartida, aquelas que obtiveram amamentação predominantemente natural $(n=109)$ não apresentavam bruxismo. $\mathrm{Da}$ mesma maneira estudos como o de Serra-Negra et al.(1997)indicam que aquelas crianças que nunca receberam aleitamento materno ou, se o fizeram, por um período de até um mês, apresentam uma chance de desenvolver hábitos deletérios sete vezes superior $(\mathrm{OR}=$ 7.1).

As maloclusões têm sido associadas com vários agentes etiológicos, tais como alergia respiratória, dieta macia, perda prematura de dentes decíduos, ausência de amamentação e respiração bucal. A etiopatogenia das maloclusões é multifatorial, interagindo fatores congênitos, morfológicos, biomecânicos e ambientais como a respiração bucal, deglutição e fonação atípica, amplamente conectados com a amamentação. Para tanto, no presente estudo observou-se associação estatisticamente significante entre o tipo de amamentação e mordida aberta anterior.Evidências transversais(LEITE et al., 1999) verificaram maior freqüência de mordidas abertas ou cruzadas entre as crianças que iniciaram precocemente o uso de mamadeira, mesmo na alimentação mista. Assim como outros estudos demonstraram ser a sucção não nutritiva possível responsável pelo surgimento de maloclusões na infância, principalmente mordida aberta anterior, overjet e mordida cruzada posterior(MOYERS, 1991).
Estudos atuais tem demonstrado que a amamentação natural pode ter efeito protetor contra o surgimento de doenças respiratórias atópicas como por exemplo, asma atópica(MIŠAK, 2011).Em contrapartida, neste estudo, não foi encontrada relação estatisticamente significante entre o tipo de aleitamento fornecido ao bebê e surgimento de alergias. Do mesmo modo, a composição do leite humano é heterogênea e sofre influências de inúmeras outras condições ambientais maternas e/ou gestacionais, podendo, muitas vezes,não proporcionar efeito protetor à algumas alterações sistêmicas(COUTINHO; SILVA, 1996; VITOLO, 1994).

Valdés e Nunes (2004)relacionaram tempo de amamentação com presença de hábitos e instabilidade psicológica da criança, conceituada como ansiedade, sono instável e choro excessivo. Seus resultados mostraram que $75 \%$ dos filhos de mães que amamentaram por menos de 3 meses apresentaram hábitos deletérios na infância, dos quais o mais prevalente foi a chupeta (57\%), 75\% dos filhos de mães que amamentaram por volta de 6 meses não apresentaram tais hábitos, um tempo menor do que 3 meses de amamentação gerou crianças ansiosas e um tempo igual ou superior a 6 meses gerou crianças com menos hábitos $e$ emocionalmente mais estáveis. Entretanto, neste estudo não foi constatado associações entre o período de amamentação e a presença de hábitos de bruxismo, respiração oral, mordida aberta anterior e alergias. Este fato pode ser observado em razão de os resultados serem obtidos por prontuários clínicos, que, por sua vez, são dependentes do conhecimento dos pais ou responsáveis sobre possíveis alterações da criança que freqüentemente possam passar despercebidas e, portanto, não são relatadas corretamente aos profissionais.

Com base no exposto, sugere-se que os profissionais da saúde que ofertam atendimento a crianças dessa faixa etária busquem o conhecimento multidisciplinar sobre o desenvolvimento infantil, uma vez que são capazes de orientar os responsáveis das crianças sobre os benefícios do aleitamento materno com o propósito de aprimorar a qualidade de vida dos pequenos, promovendo a saúde.

\section{Conclusão}

- A maioria das crianças atendidas nos ambulatórios odontológicos UNIFRA recebeu amamentação natural (85\%);

- A amamentação artificial foi associada a hábitos de respiração bucal, bruxismo e mordida aberta anterior;

- Não foi observada associação entre o tipo de amamentação (natural/artificial) e processos respiratórios alérgicos;

- Não houve relação associação entre o tempo de amamentação e a presença de hábitos de respiração oral, bruxismo, mordida aberta anterior e alergias respiratórias.

\section{Referências}

ARAÚJO, R.S; LEITÃO, R.S.S; GRINFELDE, S. Autodiagnóstico da respiração versus amamentação.International journal of dentistry, v.3, n. 1,p. 289-296, 2004.

BALDRIGHI, S.E.Z.M et al. A Importância do Aleitamento Natural na Prevenção de Alterações Miofuncionais e Ortodônticas. Dental Press Ortodon Ortop Facial, v. 6, n. 5, p. 111-21, 2001.

CARVALHO, G.D. Amamentação: Uma Avaliação Abrangente II. R Secret Saúde, v. 4, n. 28, p. 8-10, 1997. 
COUTINHO, S.B; SILVA, A.S. Aleitamento materno. In:SILVA, A. S. et al. Temas de neonatologia. Recife: Universitária. UFPE, 1996. p.128-131.

CZERNAY, A. P. C.; BOSCO, V. L. A Introdução Precoce e o Uso Prolongadoda Mamadeira: Ainda uma Realidade.J Bras Odontoped Odontol Bebê, v. 6. n. 30, p. 138-44, 2003.

FERREIRA, M.I.D; TOLEDO, O.A. Relação entre tempo de aleitamento materno e hábitos bucais. Rev ABO Nac, v.5, n.6, p.317$20,1997$.

FREITAS, F. C. N. et al. Evaluation of the plate dimensions of patientes with perenial allergic rhinitis. Int J Pediatr Dent, v.11, n.5, p.365-371, 2001.

GUERRA, M.E; MUJICA, C. Influencia del amamantamiento en el desarrollo de los maxilares. Acta Odontol Venez,v.37, n.2, p.6-10, 1999.

LEITE, I.C.G et al. Associação entre aleitamento materno e hábitos de sucção não-nutritivos.Rev Ass Paul Cir Dent, v.53, n.2, p. 151-5. 1999.

LUSVARGHI, L. Identificando o Respirador Bucal. Rev Ass Paul Cir Dent, v. 53, n. 4, p. 265-274, 1999.

MARCHESAN, I. Q. Avaliação e Terapia dos problemas da respiração. In: Marchesan I. Fundamentos em Fonoaudiologia Aspectos Clínicos da Motricidade Oral. Rio de Janeiro: Guanabara Koogan, 1998. p. 22-36.

MIŠAK, Z. Infant nutrition and allergy. Proc Nutr Soc., v.70, n. 4,p. 465-71, 2011.

MIZUNO, K.; FUJIMAKI, K.; SAWADA, M. Sucking Behavior at Breast During the Early Newborn Period Affects Later Breast-feeding Rate and Duration of Breast-feeding. Pediatr Dent, v. 46, n.1, p.15-20, 2004.

MOHEBBI, S. Z. et al. Feeding Habits as Determinants of Early Childhood Caries in a Population Where Prolonged Breastfeeding is the Norm. Community Dent Oral Epidemiol, v. 36, n.4, p.363-369, 2008.

MOREIRA, M; CORRÊA, M.S.N.P . Desenvolvimento Anatomofuncional da Boca: da Fase Pré-natal aos 3 Anos de Idade. In: CORREIA, M. S. N. P. Odontopediatria na Primeira Infância. São Paulo: Liv. Santos. 1998. cap.10, p. 101-115.

MOYERS, R. E. Ortodontia. 4a Ed. Rio de Janeiro: Guanabara Koogan; 1991

PERES, K.G et al. Social and Biological Early Life Infl uences on the Prevalence of Open Bite in Brazilian 6-years-old. Int J PaediatrDent, v. 17, n.1, p. 41-9, 2007

PRAETZEL, J. R. Distúrbios Miofuncionais da Face (DMF): um novo paradigma de atuação para a Odontopediatria. J Bras Odonto Odontoped Bebê, v. 1, n 4, p. 97-105, 1997.

PRAETZEL, J. R. et al. A Importância da Amamentação no Seio Maternopara a Prevenção de Distúrbios Miofuncionais da Face. PróFono: R Atual Cient, v. 9, n. 2, p. 69-73, 1997.
PRAETZEL, J. R. et al.Relação entre o Tipo de Aleitamento e o Uso de Chupeta.J. Bras Odontoped Odontol Bebê, v. 5, n. 25, p.23540, 2002.

PRATES, N. S., MAGNANI, M. B. B. A. e VALDRIGHI, H. C. Respiração bucal e problemas ortodônticos - causas e efeitos. Rev Paul Odontol, v.19, n.4, p. 14-9, 1997

QUELUZ, D.P; GIMENEZ, C.M.M. A síndrome do respirador bucal. Rev CROMG, v.6 n.1, p.4-9, 2000.

SCAVONE JUNIOR, $\mathrm{H}$. et al. Association between Breastfeeding Duration and Non-nutritive Sucking Habits. Community DentHealth, v. 25. n.3, p. 161-5, 2008.

SERRA-NEGRA, J. M. C. et al.Estudo da associação entre aleitamento, hábitos bucais e maloclusões. Rev Odontol Univ São Paulo, v.11, n.2, p.79-86, 1997.

TOLLARA, M. N et al. Aleitamento Natural na Primeira Infância.In: CORREA, M. S. N. P. Odontopediatria na primeira infância. São Paulo: Santos, 1998. p:71-86.

VALDES, Z.R.P; NUNEZ, D.R. Prevalência de habitos deformantes y anomalías dentomaxilofaciales en niños de 3 a 6 años de edad. Rev Cubana Estomatol, v.41, n.2, p. 7-12, 2004

VÁZQUEZ-NAVA, F. et al.Allergic Rhinitis, Feeding and Oral Habits, Toothbrushing and Socioeconomic Status. Caries Res, v. 42, n. 2, p. 141-7, 2008.

VINHA, V. H. P. O livro da Amamentação. São Paulo: CLR Balieiro, 2002.

VITOLO, M. R. Fatores que interferem na composição do leite materno. Pediatria Moderna. v. 30, n. 3, p.388-90,1994. 